\title{
NO EFFECT OF MUSCLE FIBER TYPE ON MECHANICAL EFFICIENCY DURING CYCLE EXERCISE AT $1.5 \mathrm{HZ}$
}

\author{
J. I. Medbø \\ National Institute of Occupational Health, Oslo, Norway
}

\begin{abstract}
The mechanical efficiency has been determined for 23 healthy young men to study possible effects of muscle fiber type on efficiency during cycle exercise. Each subject cycled for $10 \mathrm{~min}$ at in average 19 different powers ranging from $\approx 1.0$ to $4.6 \mathrm{~W} \mathrm{~kg}^{-1}(70-370 \mathrm{~W})$ while the pedalling frequency was kept constant at $1.5 \mathrm{~Hz}$. The rate of energy release was determined from the steady state $\mathrm{O}_{2}$ uptake measured near the end of each $10 \mathrm{~min}$ exercise period. Delta efficiency was taken as the inverse of the slope of regression of $\mathrm{O}_{2}$ uptake on power $\left(\mathrm{d} P / \mathrm{d} n \mathrm{O}_{2}\right)$. Gross efficiency at $3 \mathrm{~W} \mathrm{~kg}^{-1}$ was established, and finally, the efficiency was taken from each subject's slope of $\mathrm{O}_{2}$ uptake versus power using a common, fixed $Y$-intercept. Muscle biopsies were taken from the lateral portion of the knee extensor muscle, and muscle fibers were classified as type I or type II. The proportion of type I fibers was $0.50 \pm 0.13$ (mean $\pm s$ ), delta efficiency was $0.262 \pm 0.010$, and gross efficiency was $0.213 \pm 0.005$. There was no significant correlation between any efficiency measure and the proportion of type I fibers. A two-sided $95 \%$ confidence interval on the data suggests that if the efficiency of the two fiber types differed, the difference was less than $12 \%$. For the same subject the efficiency did not differ more than a few percents between low powers where type II fibers may be little engaged and high powers where both fiber types are active. The data therefore support the idea that the efficiency does not differ between type I and type II fibers during cycling at $1.5 \mathrm{~Hz}$.
\end{abstract}


Key words: bicycle ergometry tests, mechanical efficiency, muscle fiber types, oxygen uptake, power, statistical analysis, submaximal exercise

\section{INTRODUCTION}

During exercise chemical energy is converted to mechanical work in active muscle fibers. The ratio between the work done and the energy released to do the work is defined as the mechanical efficiency. There are two main fiber types in human skeletal muscle, but there is no general agreement about the efficiency of each fiber type. The process of shortening at the molecular level is the same: A muscle carries out work by cross-bridge cycling [19]. Since one cross-bridge cycle requires one ATP-molecule apparently irrespective of muscle fiber type, it is conceivable that the two main fiber types work equally efficiently. That is the working hypothesis for this study.

During exercise lasting more than $1 \mathrm{~h}$ and carried out at moderate intensities there is a gradual recruitment of type II fibers in the quadriceps muscle as judged from changes in glycogen concentration in single fibers [13, 14, 43, 44]. Despite this, there was no increase in the $\mathrm{O}_{2}$ uptake [44], which suggests that type II fibers worked as efficiently as type I fibers do during cycling. Different studies relating mechanical efficiency to the proportion of muscle fiber types in the knee extensor muscle have on the other hand suggested either that type II fibers work more efficiently than type I fibers do at high pedalling rates [38], that both fiber types work equally efficiently [5], or that type I fibers work more efficiently than type II fibers do [8]. In one study type I fibers appeared more efficient than type II fibers or there was no difference between the fiber types depending on the pedalling rate chosen [15]. Thus, the effect of muscle fiber type on mechanical efficiency during cycle exercise is not established. One problem is how the efficiency is determined. First, the experimental conditions were not well standardized in the studies above and may have varied systematically between subjects with different proportions of fiber types. Moreover, it requires quite extensive experiments to obtain reliable relationships between the steady state $\mathrm{O}_{2}$ uptake and exercise intensity [31]. Thus, inadequate experimental approaches may have caused the inconclusive results mentioned above. 
Further research is needed. The efficiency during cycle exercise has been related to the proportion of type I fibers in an active muscle. Since results of former studies may have been influenced by experimental shortcomings, care was taken concerning the experimental design. Since type II fibers may not be active below a certain recruitment threshold, that threshold was estimated for each subject. The relationships between $\mathrm{O}_{2}$ uptake versus power were examined. A problem that has not been considered in former studies is that muscle fiber type is usually determined only in one muscle, while many muscles are working during most exercises. A re-examination of data of Johnson and coworkers [21] shows that classification of fibers in one muscle provides essential information on fiber type proportions in other muscles too (see the appendix). Preliminary results from part of the study have been presented elsewhere [27].

\section{SUBJECTS AND METHODS}

\section{Subjects}

23 healthy men $25 \pm 4$ yr old (mean $\pm s$ ), $1.81 \pm 0.07 \mathrm{~m}$ tall, weighing $76 \pm 8 \mathrm{~kg}$ and with a maximal $\mathrm{O}_{2}$ uptake of $40 \pm 4 \mu \mathrm{mol} \mathrm{kg}^{-1} \mathrm{~s}^{-1}$ $\left(54 \pm 5 \mathrm{ml}_{\mathrm{STPD}} \mathrm{kg}^{-1} \mathrm{~min}^{-1}\right)$ volunteer as subjects in the studies. Most of the subjects were physically active students. The subjects underwent a medical examination and were thoroughly informed both orally and in writing about the purpose of the experiments and its practical details before they gave their written consent to participate. The data in this study are taken from larger series of experiments that have been approved by the institute's ethics committee or by the Ethics Committee of Health Region 2 in Norway.

\section{Procedures}

The exercise was done on a Krogh-type cycle ergometer [24], modified with modern electronic control and recordings, and all exercise was carried out at a pedalling frequency of $1.5 \mathrm{~Hz}$. This ergometer adjusts the breaking force to equal the force of gravity of a preset load placed on one side of a balance. The frequency was continuously shown to the subjects on an analog instrument, and the actual power during $10 \mathrm{~min}$ exercise deviated from the preset by $<0.1 \mathrm{~W}$ 
$(\approx 0.05 \%)$. The ergometer was equipped with drop handle bars and pedals without foot straps. Consequently work on the pedals could only be done during the downstroke.

Individual relationships between power and the $\mathrm{O}_{2}$ demand (rate of energy release expressed in $\mathrm{O}_{2}$ units) were established by measuring the steady state $\mathrm{O}_{2}$ uptake from 8 to $10 \mathrm{~min}$ of exercise at a constant power below the maximal $\mathrm{O}_{2}$ uptake. This was done $19 \pm 9$ times (range: 9-43) for each subject at powers ranging from $30 \%$ to $\approx 90 \%$ of the maximal $\mathrm{O}_{2}$ uptake $\left(0.8-4.6 \mathrm{~W} \mathrm{~kg}^{-1}, 50-370 \mathrm{~W}\right)$. The subjects did 2-5 rides of 10 min duration each day with $\approx 5$ min rest between each ride, and within each day exercises were carried out at increasing power.

\section{METHODS}

The maximal $\mathrm{O}_{2}$ uptake was determined by the levelling-off criterion [39]. Fractions of $\mathrm{O}_{2}$ and $\mathrm{CO}_{2}$ in the expired air were measured on a Scholander gas analyzer [34] or on an automatic system $\left(\mathrm{CO}_{2}\right.$ on an analyzer from Simrad Optronics, Oslo, Norway or a CD-3A analyser with a P-61B infrared-type $\mathrm{CO}_{2}$ sensor from Applied Electrochemistry, Pittsburgh, PA, USA; $\mathrm{O}_{2}$ on an S 3A/I from Applied Electrochemistry). Gas volumes were measured in a wet, Tissot-type spirometer [41] or by an S430-A ventilation measure system with a K520C521 flow transducer (Applied Electrochemistry) while the air temperature was measured simultaneously by a thermometer. The air pressure was recorded to the nearest hectopascal by a mercury barometer calibrated against high-precision instruments at the Norwegian Institute of Meteorology. From each subject at least four muscle biopsies were taken from the mid portion of the lateral portion of the knee extensor muscle ( $m$. vastus lateralis), and 200 fibers or more in each biopsy were classified as type I or type II to obtain the subjects' proportion of each fiber type in that muscle. This protocol fulfills recommendations for obtaining reliable estimates of the proportion of each fiber type in a muscle $[4,10,26]$.

The muscle fibers were classified by a histochemical method where serial sections of the biopsies were stained for myosin ATP-ase 
activity after different pre-incubations as described in detail elsewhere $[42,44]$. For 13 of the subjects type II fibers were also subclassified histochemically as type IIA, type IIAB, or type IIB according to a system worked out at this institute [42]. Fibers here called type IIB probably corresponds to those usually called human type IIX now, and likewise, those here called type IIAB corresponds to those now called type IIAX by most researchers. Since a formal comparison of the institute's method with more recent and common classifications has not been carried out, the older naming is used in this paper.

\section{Calculations and statistics}

Efficiency measures. For each subject a relationship between steady state $\mathrm{O}_{2}$ uptake and power was established by linear regression. The delta mechanical efficiency was calculated from the inverse of the regression's slope, that is from $\mathrm{d} P / \mathrm{d} n \mathrm{O}_{2}$, where $\mathrm{d} n \mathrm{O}_{2}$ is the increment in steady state $\mathrm{O}_{2}$ uptake associated with an increament $\mathrm{d} P$ in power. The $\mathrm{O}_{2}$ uptake at $3 \mathrm{~W} \mathrm{~kg}^{-1}$ was estimated from each subject's regression line, and the gross efficiency was calculated as $P / n \mathrm{O}_{2}$ where $P$ is the power of $3 \mathrm{~W} \mathrm{~kg}^{-1}$ and $n \mathrm{O}_{2}$ is the $\mathrm{O}_{2}$ demand at this power. An estimate from the regression line was preferred to a single measurement since that reduced the effect of errors in single measurements from $>0.7 \mu \mathrm{mol} \mathrm{kg} \mathrm{k}^{-1} \mathrm{~s}^{-1}$ to $0.25 \pm 0.09 \mu \mathrm{mol} \mathrm{kg}^{-1} \mathrm{~s}^{-1}$, that is, by a factor of three. The relative error of the slope and consequently also the gross efficiency was $0.008 \pm 0.003$. A third, robust measure of the efficiency was taken as follows: The $Y$-intercept varies between subjects, but the variation is probably largely of random nature [29]. An error in the $Y$-intercept will affect the slope too since the two parameters are usually (negatively) correlated [45]. Thus, an erroneously large (small) $Y$-intercept is associated with an erroneously small (large) slope that again will affect the calculated efficiency. A fixed $Y$-intercept may give more reliable relationships between $\mathrm{O}_{2}$ demand and exercise intensity [29]. Therefore, the mean of the subjects' $Y$-intercept was chosen, and for each subject a linear regression of steady state $\mathrm{O}_{2}$ uptake on power was calculated using this fixed $Y$-intercept [6]. A second measure of the delta efficiency was taken from the slope so obtained for each subject, that is as $\mathrm{d} P / \mathrm{d} n \mathrm{O}_{2}$ as above. 
The respiratory exchange ratio during the submaximal $10 \mathrm{~min}$ pretests averaged 0.93 and varied little between subjects and experiments. One mole of $\mathrm{O}_{2}$ was consequently set equal to $465 \mathrm{~kJ}$ [25].

Estimation of recruitment threshold of type II fibers. Type I fibers seem to be recruited before type II fibers [13, 14, 43, 44] in agreement with Henneman's size principle [17]. Data on glycogen depletion in single muscle fibers have shown that the proportion of muscle fibers in the lateral portion of the knee extensor muscle recruited during cycling may equal the exercise intensity relative to maximal $\mathrm{O}_{2}$ uptake [43]. The latter observation means that a subject with $40 \%$ or $70 \%$ of type I fibers recruits few or no type II fibers in that muscle during the first $20 \mathrm{~min}$ of exercise, as long as the exercise intensity is less than $40 \%$ or $70 \%$, respectively, of maximal $\mathrm{O}_{2}$ uptake. At higher intensities all type I and in addition a significant part of type II fibers in the knee extensor muscle are recruited from the onset of exercise (see Fig. 5 in Ref [43]). The recruitment threshold of type II fibers may therefore differ between subjects with different proportions of type II fibers. This finding has been used to estimate individual recruitment thresholds for type II fibers, and a separate examination showed that this estimate has a statistical error of $0.3 \mathrm{~W} \mathrm{~kg}^{-1}(25 \mathrm{~W}$, corresponding to $9 \%$ of the maximal $\mathrm{O}_{2}$ uptake). For subjects with enough measurements of $\mathrm{O}_{2}$ uptake versus power above or below the estimated thresholds according to [31], separate regressions were calculated, and the slopes so obtained were compared with those of the full data set.

Statistics. Data are given as individual results or as mean $\pm s$ (standard deviation, SD). Correlations between two variables have been expressed by the common Pearson's correlation coefficient $(r)$. Linear regressions were calculated by common least square estimators of the slope and $Y$-intercept or by using a fixed $Y$-intercept [6]. The scatter around the regression line $\left(s_{Y \mid x}\right)$ has been used as a measure of the goodness of the fit, and in addition the error of the slope $\left(s_{b}\right)$ is used. A possible breakpoint in each subject's relationship was examined as proposed by Jones and Molitoris [22]. Statistical tests were carried out in a spreadsheet, and all tests were carried out twosided since different studies have suggested that type II fibers may work either more efficiently or less efficiently that type I fibers do. 


\section{RESULTS}

\section{$\mathrm{O}_{2}$ uptake versus power. Delta mechanical efficiency}

The steady state $\mathrm{O}_{2}$ uptake rose almost linearly with power at all submaximal intensities above $\approx 1 \mathrm{~W} \mathrm{~kg}^{-1}(\approx 70 \mathrm{~W}$; Figure 1$)$. The slope was $8.2 \pm 0.3 \mu \mathrm{mol} \mathrm{J}{ }^{-1}$, which corresponds to a delta efficiency of $0.262 \pm 0.010$. The error of the slope $\left(s_{b}\right)$ was $0.22 \pm 0.07 \mu \mathrm{mol} \mathrm{J}{ }^{-1}$. This corresponds to an error of 0.007 in the efficiency or $<3 \%$ of the mean of 0.262 in single determinations of the mechanical efficiency.

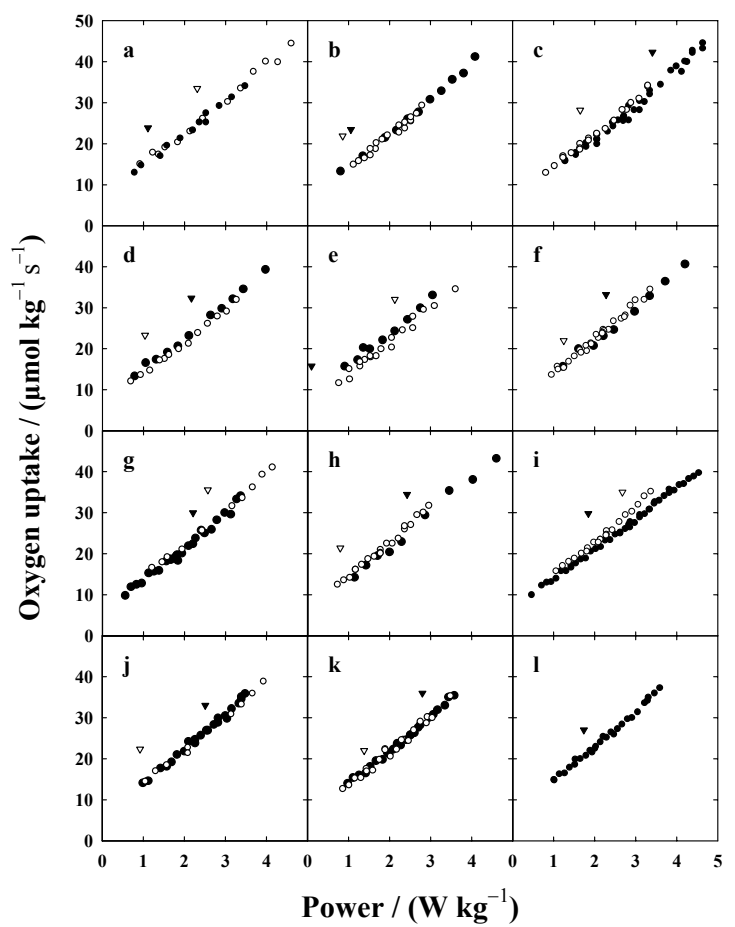

Figure 1. Steady-state $\mathrm{O}_{2}$ uptake versus power for 23 subjects cycling repeatedly for $10 \mathrm{~min}$ at different powers. The subjects had different proportions of type I fibers in their knee extensor muscle. Type I fibers are probably recruited before type II fibers, and thresholds of recruiting type II fibers estimated as explained in the methods, are shown for each subject by the triangles. 
The $Y$-intercept of the regression lines was $5.6 \pm 0.9 \mu \mathrm{mol} \mathrm{kg} \mathrm{k}^{-1} \mathrm{~s}^{-1}$ (around twice the resting $\mathrm{O}_{2}$ uptake in a supine position). The statistical error in a single estimated $Y$-intercept averaged $0.5 \mu \mathrm{mol} \mathrm{kg}{ }^{-1}$ $\mathrm{s}^{-1}$. The error of regression (scatter around the regression lines, $s_{Y \mid x}$ ) was $0.7 \pm 0.2 \mu \mathrm{mol} \mathrm{kg}^{-1} \mathrm{~s}^{-1}$, and the correlation coefficient was 0.995 \pm 0.003 .

\section{Efficiency at low and high powers}

Type II fibers may be little active at low powers [14, 40, 43]. A threshold for recruitment of type II fibers was therefore estimated for each subject as explained in the method section, and the estimated thresholds are shown by the triangles in Figure 1. The estimated threshold appeared at $1.8 \pm 0.8 \mathrm{~W} \mathrm{~kg}^{-1}$ and ranged between 0.1 and $3.4 \mathrm{~W} \mathrm{~kg}^{-1}$. There were minimal differences in efficiency between low and high powers. First, a two-line approximation did not give significantly better fits to the data $(P=0.25)$. Moreover, the slope of regression lines above the estimated threshold was $1.04 \pm 0.03$ $(n=13)$ of the slopes for all measurements for each subject with enough measurement to allow calculation of reliable relationships above the estimated threshold according to proposed directions [31]. A corresponding analysis of data below the threshold on six subjects gave a ratio of $0.97 \pm 0.03$. This analysis suggests that for the same subject the mechanical efficiency did not differ much between low powers where only type I fibers were presumably working and at higher powers where both type I and type II fibers were working (see the arrows in Figure 1). In line with this, pairs of subjects with widely different proportions of type I fibers in the knee extensor muscle had similar mechanical efficiencies (Figure $1 \mathrm{a}, \mathrm{c}-\mathrm{f}, \mathrm{h}, \mathrm{j}, \mathrm{k}$ ).

It could be argued that not all relationships were strictly linear. For example, that of subject RN (filled symbols in Figure 1c) shows a slight convex curvature. Second-order curve fits were therefore also examined. These fits reduced the error of regression by $0.10 \pm 0.10 \mu \mathrm{mol} \mathrm{O} \mathrm{kg}^{-1} \mathrm{~s}^{-1}\left(14 \%\right.$, from 0.7 to $\left.0.6 \mu \mathrm{mol} \mathrm{O} \mathrm{kg}^{-1} \mathrm{~s}^{-1}\right)$. However, for four subjects second-order fits actually increased the error of regression slightly. Moreover, both the variability and error in the estimated $Y$-intercepts rose by a factor of 2.5. Finally, simulations where single measurements of the $\mathrm{O}_{2}$ uptake were excluded changed the outcome of second-order fits considerably, showing that these fits were not numerically stable. 


\section{Efficiency versus muscle fiber type}

The proportion of type I fibers in the lateral portion of the knee extensor muscle was $0.50 \pm 0.13$, and the error in the single determination of fiber type proportion was 0.036 . There was no statistically significant relationship between proportion of type I fibers in this muscle and mechanical efficiency taken from the slope (delta efficiency, $r=-0.36 ; P=0.09$; Figure 2 a). However, the negative correlation means that subjects with a high proportion of type 1-fibers tended to work less efficiently than subjects with less type I-fibers as judged from the delta efficiency.

Two other and presumably more robust measures of efficiency were also calculated and related to the proportion of type I fibers. First, the gross efficiency of $0.213 \pm 0.005$ at a power of $3 \mathrm{~W} \mathrm{~kg}^{-1} \mathrm{did}$ not correlate significantly to the proportion of type I fibers in the knee extensor muscle ( $r=0.26, P=0.24$; Figure $2 \mathrm{~b}$ ).

Denoting the correlation coefficient $r$, the $Y$-intercept $Y_{0}$ and the slope $b$, the correlation $r\left(Y_{0}, b\right)=-0,94$ for the present data calculated according to [45], and for all individual correlations $r_{i}\left(Y_{0}, b\right)$ $<-0,9$. Thus, an erroneously large (small) $Y$-intercept was associated with an erroneously small (large) slope, which again will affect the delta efficiency. The individual $Y$-intercepts did not vary much more than what can be ascribed to the statistical error in single estimates, which is compatible with the idea that the observed differences in $Y$-intercepts between subjects were largely of random origin. To minimize random effects on the calculated delta efficiency and thus to get another robust efficiency measure, the slope of regression of $\mathrm{O}_{2}$ uptake on power was calculated using a fixed $Y$-intercept of $5.6 \mu \mathrm{mol} \mathrm{kg} \mathrm{kg}^{-1}$ (the mean for the 23 subjects). The slope was still $8.2 \pm 0.3 \mu \mathrm{mol} \mathrm{O}_{2} \mathrm{~J}^{-1}$, and the error of regression was $0.7 \pm$ $0.2 \mu \mathrm{mol} \mathrm{kg} \mathrm{kg}^{-1} \mathrm{~s}^{-1}$ (unchanged), but the error of the slope was reduced to $0.08 \pm 0.03 \mu \mathrm{mol} \mathrm{J} \mathrm{J}^{-1}$, that is by $64 \%$. The efficiency calculated this way was still $0.262 \pm 0.010$, but the error in single efficiencies was now 0.0025 or less than $1 \%$ of 0.26 . That efficiency measure did not correlate to the proportion of type I fibers $(r=0.30, P=0.17$; Figure 2 c). 

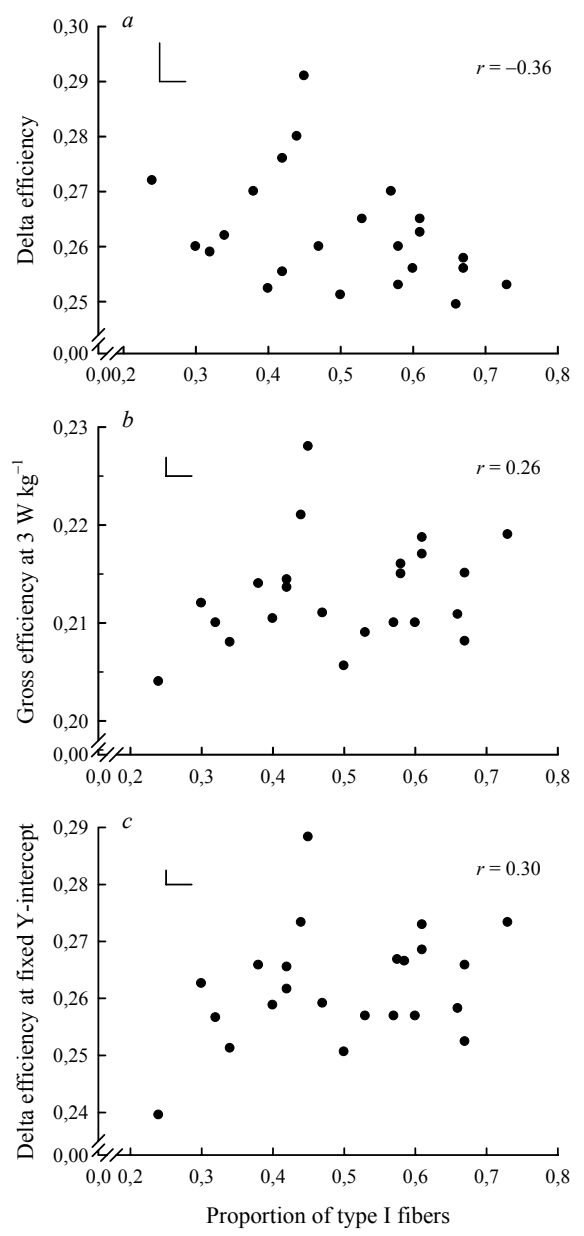

Figure 2. Mechanical efficiency versus proportion of type I fibers in the lateral portion of the knee extensor muscle. $a$, delta efficiency versus fiber type proportion; $b$, gross efficiency at $3 \mathrm{~W} \mathrm{~kg}^{-1}$ versus fiber type proportion; $c$, delta efficiency using a common, fixed $\mathrm{Y}$-intercept versus fiber type proportion. The data are from 23 subjects who cycled repeatedly for $10 \mathrm{~min}$ at different powers while the steady state $\mathrm{O}_{2}$ uptake was measured near the end of exercise. The right-angle mark (L) in each panel shows the statistical or analytical error $(s)$ in single determinations of fiber type proportion and efficiency, respectively. 
Type II fibers were further subclassified for 13 subjects, and it appeared that $62 \%$ of the type II fibers were of type IIA $(33 \%$ of all fibers), $10 \%$ were of type IIAB ( $5 \%$ of all), and $28 \%$ were of type IIB ( $15 \%$ of all). It should be noted that only eight subjects had more than $10 \%$ of type IIB fibers. The proportion of type IIB-fibers correlated positively to the delta efficiency $(r=0.57 ; P=0.04)$ but not to the other two and more robust efficiency measures $(|r| \leq 0.07 ; P>0.8)$.

\section{Extrapolation to zero and hundred percent of type I fibers}

To estimate the efficiency of type I and type II fibers relationships of efficiency versus fiber type proportion may be extrapolated to zero and hundred percent type I fibers (or type II fibers). Three different efficiency measures gave somewhat different correlations to the proportions of type I fibers. Moreover, an examination of the data shows that for one extreme subject (SRS) with only $24 \%$ type I fibers the position relative to the other subjects varied between $1 \mathrm{SD}$ above the average (delta efficiency, Figure 2 a) to around 2 SD below the average (gross efficiency and delta efficiency using a common $Y$-intercept; Figure $2 \mathrm{~b}$ and $\mathrm{c}$; see the leftmost point in each panel in Figure 2). The relationships for this subject were based on only nine measurements of steady state $\mathrm{O}_{2}$ uptake and is thus less reliable than those for most of the other subjects. Recalculating the relationships after excluding the data of SRS gave correlations $r \leq 0.12(P=0.6)$ for gross efficiency and delta efficiency using a fixed $Y$-intercept to the proportion of type I fibers. Exclusion of the data of SRS had less effect on the correlation of delta efficiency to proportion of type I fibers $(r=-0.31 ; P=0.16)$.

Therefore, in further analyses the slope of regression of efficiency on proportion of type I fibers was set to zero, corresponding to zero correlation. The error of the slope of regression of delta efficiency using a common, fixed $Y$-intercept on proportion of fiber type was 0.0154 and somewhat less for the other two efficiency measures. Using this value for the error of the slope gives the interval $[-0.032$, +0.032 ] as a two-sided $95 \%$ confidence interval around a slope of zero. Extrapolating the upper end of the confidence interval to zero and $100 \%$ type I fibers gives efficiencies of 0.246 ( $0 \%$ type I fibers) and 0.278 (100\% type I fibers). The ratio of these two values is 0.88 . A corresponding extrapolation of the lower end of the confidence interval or for zero and $100 \%$ type II fibers gives the same result. Thus, the data suggest that if the efficiency of the two main fiber types 
differed in the present study, the difference is no more than $12 \%$. These calculations were repeated using data of the other two efficiency measures. Those calculations gave somewhat narrower confidence intervals.

\section{DISCUSSION}

The delta efficiency averaged 0.26 while the gross efficiency at a power of $3 \mathrm{~W} \mathrm{~kg}^{-1}$ was 0.21 . The efficiency varied between subjects, but it was not related to the proportion of type I fibers in the knee extensor muscle. Delta efficiency did not differ much between low and high powers despite a probably larger contribution from type II fibers at high powers. Finally, a statistical evaluation of the data suggests that if the two fiber types differ in their mechanical efficiency, the difference is no more than $12 \%$ during cycling at $1.5 \mathrm{~Hz}$.

\section{Methodological considerations of mechanical efficiency}

Former studies addressing the efficiency during exercise have not standardized their conditions adequately, and that may have influenced their results. Therefore some methodological issues on establishing the efficiency are addressed. The efficiency has in this study been determined for each subject based on measurements of the $\mathrm{O}_{2}$ uptake during 8-10 min of exercise at constant intensity since a steady state is usually reached within 8 min of exercise $[1,9,23]$. Exercise was done during conditions where anaerobic energy release is negligible [32]. Thus, the measured $\mathrm{O}_{2}$ uptake reflects the total ATPturnover rate. The efficiency was determined individually from at least nine and in average 19 values of the steady state $\mathrm{O}_{2}$ uptake measured at a wide range of powers. The approach used is thus in line with recommendations for obtaining reliable relationships that suggest a minimum of eight measurements [31].

By carrying out the experiments along the principles outlined above, the statistical error in single determinations of delta efficiency was only 0.007 or less than $3 \%$ of the mean value of 0.26 . By using a fixed $Y$-intercept the error was reduced further to less than $1 \%$. The gross efficiency was determined from the established regression lines rather than from single measurements since that reduced errors in single determinations from 0.005 to $<0.002$, that is from $2.5 \%$ to 
$0.8 \%$ of the mean value of 0.21 . Thus, errors in single efficiencies were far less than those in other studies. Moreover, the errors were also much less than the between-subjects-variations. This means that it is not likely that analytical and statistical errors have masked a systematic effect and thus influenced conclusions drawn from the present data.

Other studies have used only one or a few measurements for establishing the relationship between exercise intensity and $\mathrm{O}_{2}$ uptake $[5,8,15]$, which makes their data less reliable. In some studies the measurements used cover a quite narrow range [8, 38], and that increased the imprecision in each delta efficiency determined.

There is a disproportionately high $\mathrm{O}_{2}$ uptake at low exercise intensities that will introduce nonlinear effects at low powers (see Figures in Ref. [28, 31]), a phenomenon that may have a simple biomechanical explanation. Exercise at intensities below $\approx 1 \mathrm{~W} \mathrm{~kg}^{-1}$ was therefore avoided, and thus no essential nonlinear effects were seen (Figure 1). Another study did not avoid the problem of high $\mathrm{O}_{2}$ uptake at low powers for the group with mainly type II fibers [38], and that may have influenced the conclusions drawn in that study.

The gross efficiency was taken from the same quite high power for all subjects. That is important as the following calculations show. Assume that the $\mathrm{O}_{2}$-uptake $\left(n \mathrm{O}_{2}\right)$ rises linearly with power $(P): n \mathrm{O}_{2}=$ $A+b P$ where $A$ is the $Y$-intercept and $b$ the slope. The gross efficiency is then $\eta_{\text {gross }}=P / n \mathrm{O}_{2}$, and its inverse, a measure of the energetic cost of a standardized work or exercise intensity, is $\left(\eta_{\text {gross }}\right)^{-1}=$ $n \mathrm{O}_{2} / P=(A+b P) / P=A / P+b$. Since all subjects were compared at the same power $P$, the inverse gross efficiency $\left(\eta_{\text {gross }}\right)^{-1}$ is the slope $b$ plus a nearly constant term $A / P$ since the $Y$-intercept $A$ did not vary much between subjects and $P$ was fixed at $3 \mathrm{~W} \mathrm{~kg}^{-1}$. If on the other hand $P$ differs between subjects, then $\left(\eta_{\text {gross }}\right)^{-1}$ and thus also $\eta_{\text {gross }}$ will vary accordingly, resulting in higher apparent gross efficiencies the higher the power. Three studies that found a relationship between fiber type proportion and efficiency, did not standardize the experimental conditions sufficiently $[8,15,38]$. Bosco and coworker who found no relationship between fiber type proportion and efficiency, examined their subjects at the same power [5].

It could be argued that inadequately controlled experimental conditions should not introduce systematic effects. That is true only if the factors not controlled for are unrelated to fiber type proportion. The 
training state may for example correlate to fiber type proportion [7], and even a statistically nonsignificant correlation may introduce a systematic effect if it is large. In line with this, it is noteworthy that the variation in efficiency between subjects in other studies $[8,15]$ was 2-3 times larger than in the present one.

Three different measures of efficiency were used here. The delta efficiency using a fixed $Y$-intercept is regarded as the best measure. When adequately established, the $Y$-intercept does not seem to vary systematically between subjects but shows variations that seem random but that nevertheless affect the calculated delta efficiency. That possibly random effect is minimized when a fixed intercept is used. In addition, the approach using delta efficiency with a fixed $Y$-intercept showed very little statistical error in single determinations in this study.

Second-order curve fits and two-line fits were also examined here. If present at all, the second-order component was small. Moreover, the relationships found were unstable and numerically nonrobust. Thus, use of second-order fits seems unreliable in the analysis of relationships between $\mathrm{O}_{2}$ uptake and exercise intensity. Likewise, two-line fits did not identify breakpoints and improve the relationships.

\section{Effect of fiber type on efficiency}

There was no effect of fiber type on efficiency in this study. An efficiency independent of fiber type working is supported by data from several other studies. During exercise at constant power lasting many minutes to more than an hour, there is a gradual recruitment of type II fibers by time in the quadriceps muscle [13, 14, 43, 44]. Despite this there is little change in the $\mathrm{O}_{2}$ uptake $[1,9,23,44]$. Moreover, as mentioned above, Bosco and coworkers who examined the efficiency for 32 subjects cycling at $1.0 \mathrm{~Hz}$, found no correlation to the proportion of type I fibers in the knee extensor muscle [5]. Contrary to other studies showing an apparent effect of muscle fiber type, Bosco and coworkers standardized their experimental conditions. The authors suggested that their finding was a consequence of the pedalling frequency of $1.0 \mathrm{~Hz}$ chosen and that an effect might be seen at higher or lower frequencies. The present study carried out at $50 \%$ higher frequency, but there was still no effect of fiber type on efficiency.

The present results are at variance with other studies that suggested either that type II fibers work more efficiently than type I 
fibers do at high pedalling frequencies [38], or that type I fiber work more efficiently than type II fibers do [8, 15]. As pointed out above, there are several problems in the experimental design in these studies that may have influenced the results.

As a further analysis of the effect of fiber type on efficiency, relationships between $\mathrm{O}_{2}$ uptake and power were determined at powers above and below the estimated recruitment threshold of type II fibers. The efficiency did not differ much between low and high powers. This analysis assumes that there is little activation of type II fibers at low powers, as suggested by measurements $[43,44]$ as well as semi-quanitative analyses $[13,14,40]$ of changes in muscle glycogen concentration in single fibers of the knee extensor muscle. These conclusions may be challenged by data of Ivy and coworkers who measured concentrations of phosphocreatine, lactate, malate, glucose6-phosphate, and ATP in single fibers of the knee extensor muscle during exercise [20]. Almost all fibers of both fiber types appeared active even at powers below $50 \%$ of the maximal $\mathrm{O}_{2}$ uptake as judged from changes in the concentration of measured muscle metabolites. However, their system may be very sensitive and pick up even the slightest activation of type II fibers, too low to break down significant amounts of glycogen and contribute considerably to the work done. A synthesis and compromise of data of the studies mentioned above may suggest that at low powers mainly but not exclusively type I fibers work, while at higher powers both fiber types contribute considerably. This interpretation is in line with data on recruitment of single motoneurons showing that small motoneurons activating type I muscle fibers are usually but not always activated before larger neurons innervating type II fibers are [18, 37]. Thus, the analysis of recruitment threshold of type II fibers as suggested in figure 1 seems largely justified.

There was no significant correlation between the proportion of type IIB fibers and the two most robust efficiency measures used. If these fibers were recruited at low and moderate powers, this suggests that even type IIB fibers may work with an efficiency similar to that of type I fibers during cycling at $1.5 \mathrm{~Hz}$. However, it may be that type IIB fibers are recruited only at quite high powers [43]. Therefore, no certain conclusion can be drawn on efficiency of type IIB fibers from the insignificant correlation found in this study. However, data of another study showed little increase in the $\mathrm{O}_{2}$ uptake as type IIB 
fibers were involved towards the end of exercise [44]. This is compatible with the idea that also type IIB fibers work as efficiently as other muscle fibers do.

\section{Theoretical considerations and muscle efficiency of in vitro studies}

Muscles work by cross-bridge cycling [19], and one cross-bridge cycle appears to require one molecule of ATP irrespective of muscle fiber type. If so, one would not expect the efficiency to vary with fiber type. That conclusion may be too simplistic since other processes in the muscle like ion pumping require ATP. The Na, K-pump may require only $\leq 2 \%$ of the total ATP-turnover during exercise $[11,30]$. If so, possible differences between fiber types may not be of quantitative importance. Removal of calcium ions from cytosol to sarcoplasmic reticulum is another ion-pumping process that requires ATP. Effects of possible differences between fiber types concerning calcium pumping are more difficult to estimate.

In the present study energy release was taken from the measured $\mathrm{O}_{2}$ uptake that rose with power. While working muscles use most of the $\mathrm{O}_{2}$, some of the extra $\mathrm{O}_{2}$ taken up during exercise is consumed by the heart and the ventilatory system. This means that the delta efficiency of $\approx 0.26$ as calculated in the present study for the whole body is less than that for the working leg or muscles. Comparable measurements on single muscle should therefore give higher values for the efficiency since these measurements are not influenced by $\mathrm{O}_{2}$ consumption of heart and respiratory muscles.

Some studies on the efficiency of rat muscles stimulated in vitro have suggested that muscle with mainly type I fibers are twice as efficient as those dominated by type II fibers [12, 16, 46]. The efficiency in those studies ranged between 0.07 and 0.18 , roughly half the efficiency in the present experiments, which means that in these studies isolated muscles appeared to require roughly twice as much energy as the present subjects did to do a certain amount of work. The low efficiency in these studies suggests that extrapolating the results to exercising humans may be questioned.

More recent studies have used different stimulation protocols and found muscle efficiencies in the range $0.3-0.5[2,16]$. The reported efficiency varies considerably between protocols used. In some experiments muscle with mainly type I fibers were more efficient than those with mainly type II fibers, while in other studies type II fibers appeared more efficient than type I fibers. Since the mechanical 
efficiency found depended on the protocol used, it is not known to what extent results from these studies can be extrapolated to exercising humans.

\section{Further methodological considerations}

The recruitment threshold of type II fibers was estimated using others data on the knee extensor muscle [43]. Other muscles work too during cycling, and these muscles may have different recruitment thresholds for type II fibers. The estimated thresholds (see arrowheads in Figure 1) should therefore be taken only as rough indicators of where recruitment of type II fibers begins. Since the proportion of type I fibers differed widely between the subjects, even these rough estimates may be of value. It should further be pointed out that the insignificant correlation between efficiency and fiber type is not affected by incorrect estimation of the recruitment threshold.

The experiments were carried out at a quite high pedalling frequency of $1.5 \mathrm{~Hz}$, and most other studies have used lower frequencies. It could be that at the frequency used type II fibers are recruited even at low powers. However, the pattern of glycogen breakdown in single muscle fibers does not differ between frequencies of 0.5 and $2.0 \mathrm{~Hz}$ [14].

For each subject the proportion of each fiber type was established from biopsies taken from only one muscle. For each subject a minimum of 200 fibers were classified as type I or type II in each of at least four biopsies from the lateral portion of the knee extensor muscle (m. vastus lateralis). This gives a good estimate of the fiber type proportion in that muscle $[4,10,26]$. The knee extensor muscle is only one of several important muscles used during cycling. Information about the muscle fiber type proportion in other working muscles is therefore needed. That issue is addressed in the appendix where a statistical analysis on others data [21] shows that the fiber type proportion in one muscle, for example the knee extensor, provides important information about the proportion of fiber types in all working muscles when different persons are compared.

\section{CONCLUSION}

Data from the present study suggest that type II fibers work as efficiently as type I fibers do during cycling at a frequency of $1.5 \mathrm{~Hz}$. 


\section{APPENDIX}

\section{Statistical analysis of fiber type proportions in $\mathbf{5 0}$ muscle sites}

Background. In most muscle biopsy experiments, samples are taken from only one muscle, while many muscles are usually working during exercise, for example during cycling. Data relating physical performance to muscle fiber type proportions in one muscle have limited relevance unless data on fiber type proportions are relevant to all active muscles. This appendix shows that there is a close relationship between the proportions of type I fibers in different muscles when different subjects are compared.

Methods. Johnson and coworkers classified and reported fiber types in biopsies from fifty sites in altogether 36 muscles in six previously healthy men 17-30 yr old suffering from sudden deaths [21]. Their data are re-analysed here. Data of altogether 290 samples were reported (Table 2 in ref. [21]). Data on ten missing values have here been estimated according to Snedecor and Cochran [36]. A simple two-way analysis of variance (ANOVA) on these data has been carried out, using the following model:

$$
p_{i j}=\mu+s_{i}+m_{j}+e_{i j}
$$

$\mu$ is the mean proportion of type I fibers in all samples (grand mean); $s_{\mathrm{i}}, i=1, \ldots, 6$, denotes the six different subjects; $m_{j}, j=1, \ldots, 50$, denotes the fifty different muscles or muscle sites; $e_{i j}$ is a normally distributed error term with expectation $E\left(e_{i j}\right)=0$, and $p_{i j}$ is the measured proportion of type I fibers in the sample from subject $s_{i}$ on muscle site $m_{j}$. The analysis of variance was carried out in SPSS version 15.0 using a model without interactions, and the corrections required to account for ten estimated values were calculated by hand. Further calculations were carried out in a spreadsheet. The relative error in estimated SDs from $n$ measurements was taken as $(2 n)^{-0.5}$. 

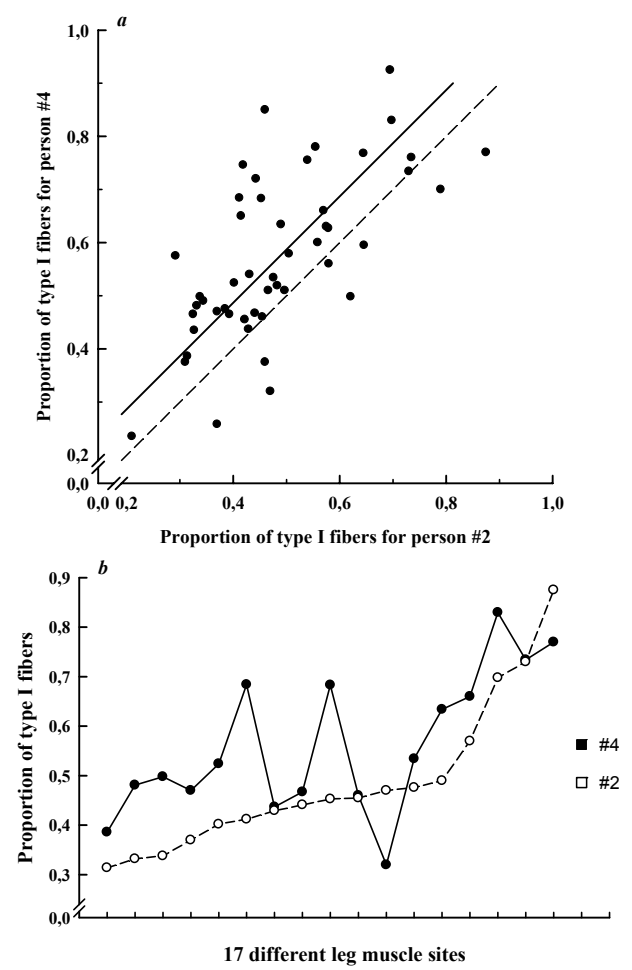

Figure 3. Biopsy data from Johnson and coworkers on proportion of type I fibers in different muscle sites [21]. Data from subjects \#2 and \#4 are used. Subject \#2 had the second smallest and subject \#4 the second largest proportions of type I fibers in the 17 leg muscle sites examined on six persons, and their data are thus used as maximum likelihood estimates of the 0.25 and 0.75 fractile in a human population. $a$, data on proportion of type I fibers in 49 muscle sites of person \#4 versus person \#2; data on $m$. frontalis were missing from person \#2. The dashed line is the line of identity, while the solid line is a parallel line displaced 0.09 , which is the difference between the mean proportion of type I fibers of 0.48 for person \#2 and of 0.57 for person \#4. $b$, biopsy data from 17 sites from nine hip, thigh, and calf muscles (person \#2, open symbols and dashed curve; person \#4 filled symbols and solid curve). The different sites are ranked in rising order of proportion of type I fibers for person \#2. The mean proportion of type I fibers in these 17 muscle sites was 0.49 for person \#2 $\left(^{\circ}\right)$ and 0.56 for person \#4 $(\bullet)$ and are shown to the right. 
Model checking. The model (A1) assumes that the error terms are normally distributed with the same variance. The following three tests show that the data followed these assumptions closely: First, the residuals were plotted on a normal probability paper and appeared linear. Second, the skewness and (corrected) kurtosis of the residuals were 0.14 and 0.3 , respectively, which differ insignificantly from the expected values of 0.0 for a normal distribution $(P=0.3)$. Third, the variance of a sample drawn from a normal distribution follows a $\chi^{2}$ distribution. Accordingly, the variance within each muscle site (across the six subjects) and the variance within each subject (across the 50 muscle sites) were plotted versus a $\chi^{2}$ distribution with the appropriate degrees of freedom (five and 49, respectively), and close linear relationships between the expected and observed values were found.

Results. The two-way ANOVA showed significant differences in the proportions of type I fibers between persons $\left(F_{5,235}=44.4\right.$; $P<0.001)$ and between different muscle sites $\left(F_{49,235}=14.5\right.$; $P<0.001)$, and the random variation term was $0.089 \pm 0.004$. For example, person \#1 who had the largest mean proportion of type I fibers among the six men $\left(\bar{p}_{\mathrm{tI}, \# 1}=0.66\right)$ had relatively more type I fibers than all the other five men in 33 out of 50 muscle sites examined. Likewise, person \#3 who had the smallest mean proportion of type I fibers $\left(\bar{p}_{\mathrm{t}, \# 3}=0.43\right)$ had the smallest proportion of type I fibers among the six men in 25 of 50 muscle sites examined. Moreover, person \#1's proportion of type I fibers was larger than that of person \#3 in all 50 muscle sites examined.

Persons \#1 and \#3 were extremes within the sample of six men and thus not suited for describing typical cases. Persons \#2 and \#4 had the second smallest and second largest, respectively, proportion of type I fibers in a subsample of 17 leg muscle sites examined further below, and these two men were therefore chosen as (maximum likelihood) estimates of the 0.25 and 0.75 fractile (first and third quartile), respectively, in a human population of healthy young men. The proportion of type I fibers was larger for person \#4 than for person \#2 in 41 out of 49 muscle sites (Figure $3 \mathrm{a} ; P<10^{-5}$ on a binomial test).

Johnson's data include a number of muscles probably little active during cycling and running. Their data from 17 leg muscle sites probably working during leg exercise were re-examined. Person \#4's proportion of type I fibers was larger than for person $\# 2$ in 15 out of 
the 17 muscle sites $(P=0.004)$ despite the residual variation of 0.076 \pm 0.006 in that subsample (Figure $3 b$ ).

The two-way analysis of variance did not allow analysis of possible interactions since there were no replications (only one value in each cell in the two-way layout). Thus, the observed residual variation is really a pooled effect of true residual variation plus a possible interactions. The latter effect is probably minimal. First, there is little reason to assume interactions here. Second, if interactions were significant, the observed residual variation would probably not appear normally distributed. Finally, the observed residual variation of $\approx 0.08$ is similar to the error of measurement of the fiber type proportion based on one muscle biopsy [4, 10, 26].

Comparisons of with other studies. The standard deviation in proportion of type I fibers between persons within muscle sites averaged 0.11 in the data of Johnson and coworkers. That value is similar to the standard deviation of 0.13 reported by Coyle and coworkers for 19 well-trained cyclists [8] and to $s_{\text {type I }}=0.13$ found in the present study too. Barstow and coworkers reported a standard deviation of 0.16 for their nine subjects [3], Pedersen and coworkers reported a standard deviation of 0.15 for their 12 subjects [33], while Simoneau and coworkers found a standard deviation of 0.14 for 50 subjects examined [35]. It should be noted that the observed standard deviation in each study is a combined effect of true differences between subjects and sampling errors. The latter term may vary between 0.03 and 0.08 $[4,10,26]$.

The average proportion of type I fibers in the deep portion of $m$. vastus lateralis was 0.47 among the men studied by Johnson and coworkers [21]. That value does not differ much from the proportion of 0.55 among the 19 well-trained cyclists of Coyle and coworkers [8], of 0.47 for nine subjects examined by Barstow and coworkers [3], of 0.54 for 50 subjects examined by Simoneau and coworkers [35] and 0.50 among the 23 moderately trained young men in the present study. The value differs considerably from the mean proportion of 0.69 found on well-trained men by Pedersen and coworkers [33]. Finally, the proportion of type I fibers in seven different sites of the knee extensor muscle average 0.44 among the men studied by Johnson and coworkers. Thus, although they examined only six young men, it is conceivable that their data are quite typical for what is seen among healthy young men in a general population. 
Conclusions. The fiber type proportion varies systematically between persons and muscles. The proportion in each subject's muscle can be described by a simple two-factor additive model (A1). This means that if two persons are examined and the proportion of type I fibers in one muscle differ by $\Delta p$ between the two persons, (A1) suggests that the difference between the two persons in the proportion of type I fibers is $\Delta p$ for all muscles, apart from the sampling error. Persons with a high proportion of type I fibers in the knee extensor muscle will thus most likely have a relative high proportion of type I fibers in all muscles.

\section{ACKNOWLEDGEMENTS}

I am grateful to Dr. Izumi Tabata and engineer Bjørg Ingrid Selberg who carried out the fiber typing.

\section{REFERENCES}

1. Bahr R., Opstad P. K., Medbø J. I., Sejersted O. M. (1991) Strenuous prolonged exercise elevates resting metabolic rate and causes reduced mechanical efficiency. Acta Physiol. Scand. 141: 555-563.

2. Barclay C. J. (1994) Efficiency of fast- and slow-twitch muscles of the mouse performing cyclic contractions. J. Exp. Biol. 193: 65-78.

3. Barstow T. J., Jones A. M., Nguyen P. H., Casaburi R. (2000) Influence of muscle fiber type and fitness on the oxygen uptake/power output slope during incremental exercise in humans. Exp. Physiol. 85: 109116.

4. Blomstrand E., Ekblom B. (1982) The needle biopsy technique for fibre type determination in human skeletal muscle - a methodological study. Acta Physiol. Scand. 116: 437-442.

5. Bosco C., Komi P. V., Sinkkonen K. (1980) Mechanical power, net efficiency and muscle structure in male and female middle-distance runners. Scand. J. Sports Sci. 2: 47-51.

6. Box G. E. P., Hunter W. G., Hunter J. S. (1978) Statistics for Experimenters. An Introduction to Design, Data Analysis, and Model Building. John Wiley \& Sons, New York. 453-462, ISBN 0-471-09315-7.

7. Coyle E. F., Feltner M. E., Kautz S. A., Hamilton M. T., Montain S. J., Baylor A. M., Abraham L. D., Petrek G. W. (1991) Physio- 
logical and biomechanical factors associated with elite endurance cycling performance. Med. Sci. Sports Exerc. 23: 93-107.

8. Coyle E. F., Sidossis L. S., Horowitz J. F., Beltz J. D. (1992) Cycling efficiency is related to the percentage of type I muscle fibers. Med. Sci. Sports Exerc. 24: 782-788

9. Dick R. W., Cavanagh P. R. (1987) An explanation of the upward drift in oxygen uptake during prolonged submaximal downhill running. Med. Sci. Sports Exerc. 19: 310-317.

10. Elder G. C. B., Bradbury K., Roberts R. (1982) Variability of fiber type distribution within human muscles. J. Appl. Physiol. 53: 14731480.

11. Evertsen F., Medbø J. I., Jebens E., Nicolaysen K. (1997) Hard endurance-training increases $\mathrm{Na}^{+}-\mathrm{K}^{+}$pump concentration in skeletal muscle of elite cross-country skiers. Am. J. Physiol. Regulatory Integrative Comp. Physiol. 272: R1417-R1424.

12. Gibbs C. L., Gibson R. (1972) Energy production of rat soleus muscle Am. J. Physiol. 223: 864-871.

13. Gollnick P., Armstrong R. B., Saubert C. W., Sembrowick C. L., Shepherd R. E., Saltin B. (1973) Glycogen depletion patterns in human skeletal muscle. Pflügers Arch. 344: 1-12.

14. Gollnick P., Piehl K., Saltin B. (1974) Selective glycogen depletion pattern in human muscle fibres after exercise of varying intensity and varying pedalling rates. J. Physiol. 241: 45-57.

15. Hansen E. A., Andersen J. L., Nielsen J. S., Sjøgaard G. (2002) Muscle fibre type, efficiency, and mechanical optima affect freely chosen pedal rate during cycling. Acta Physiol. Scand. 176: 185-194.

16. Heglund N. C., Cavagna G. A. (1987) Mechanical work, oxygen consumption, and efficiency in isolated frog and rat muscle. Am. J. Physiol. Cell Physiol. 253: C22-C29

17. Henneman E. (1957) Relationship between size of neurons and their susceptibility to discharge. Science 126 (3287): 1345-1347.

18. Henneman E., Somjen G., Carpenter D. O. (1965) Functional significance of cell size in spinal motoneurons. J. Neurophysiol 28: 560580.

19. Huxley H. E. (1985) The crossbridge mechanism of muscular contraction and its implications. J. Exp. Biol. 115: 17-30.

20. Ivy J. L., Chi M. M.-Y., Hintz C. S., Sherman W. M., Helledall R. P., Lowry O. H. (1987) Progressive metabolic changes in individual human muscle fibers with increasing work rates. Am. J. Physiol. 252: C630-C639. 
21. Johnson M. A., Polgard J., Weightman D., Appleton D. (1973) Data on the distribution of fibre types in thirty-six human muscles. An autopsy study. J. Neur. Sci. 18: 111-129.

22. Jones R. H., Molitoris B. A. (1984) A statistical method for determining the breakpoint of two lines. Anal. Biochem. 141: 287-290.

23. Kalis J. K., Freund B. J., Joyner M. J., Jilka S. M., Nittolo J., Wilmore J. H. (1988) Effect of ß-blockade on the drift in $\mathrm{O}_{2}$ consumption during prolonged exercise. J. Appl. Physiol. 64: 753-758.

24. Krogh A. (1913) A bicycle ergometer and respiration apparatus for the experimental study of muscular work. Scand. Arch. Physiol. 30: 375-394.

25. Lentner C. (ed.) (1981) Units of Measurements, Body Fluids, Composition of the Body, Nutrition. Geigy Scientific Tables, Vol. 1. CibaGeigy Inc., Basel. 228-231.

26. Lexell J., Taylor C., Sjöström M. (1985) Analysis of sampling errors in biopsy techniques using data from whole muscle cross sections. J. Appl. Physiol. 59: 1228-1235.

27. Medbø J. I. (1990) Type I and type II fibres work with the same mechanical efficiency during bicycling. In: Muscle and Motility, vol 2. Maréchal G., Carraro U. (eds.). Proceedings of the $19^{\text {th }}$ European Conference in Brussel. Intercept, Andover, Hampshire. 303-308.

28. Medbø J. I. (1996) Is the maximal accumulated oxygen deficit an adequate measure of the anaerobic capacity? Can. J. Appl. Physiol. 21: 370-383.

29. Medbø J. I. (2005) Examination of different ways to establish relationships between the $\mathrm{O}_{2}$ demand and the exercise intensity. Acta Kinesiol. Univ. Tartuensis 10: 89-115.

30. Medbø J. I., Jebens E., Vikne H., Refsnes P. E., Gramvik P. (2001) Effect of strenuous strength training on the muscle Na-K pump concentration in skeletal muscle of well-trained men. Eur. J. Appl. Physiol. 84: 148-154.

31. Medbø J. I., Mohn A.-C., Tabata I., Bahr R., Vaage O., Sejersted O. M. (1988) Anaerobic capacity determined by the maximal accumulated $\mathrm{O}_{2}$ deficit. J. Appl. Physiol. 64: 50-60.

32. Medbø J. I., Tabata I. (1989) Aerobic and anaerobic energy release and work during shortlasting, exhausting bicycle exercise. J. Appl. Physiol. 67: 1881-1886.

33. Pedersen P. K., Sørensen J. B., Jensen K., Johansen L., Levin K. (2002) Muscle fiber type distribution and nonlinear $\mathrm{VO}_{2}$-power output relationship in cycling. Med. Sci. Sports Exerc. 34: 655-661. 
34. Scholander P. F. (1947) Analyzer for accurate estimation of respiratory gases in one-half cubic centimetre sample. J. Biol. Chem. 167: $1-15$.

35. Simoneau J.-A., Lortie G., Boulay M. R., Thibault M.-C., Bouchard C. (1986) Repeatability of fibre type and enzyme activity measurements in human skeletal muscle. Clin. Physiol. 6: 347-356.

36. Snedecor G. W., Cochran W. G. (1967) Statistical Methods. Iowa State Univ. Press, Ames, IA, USA. 317-321.

37. Somjen G., Carpenter D. O., Henneman E. (1965) Responses of motoneurons of different sizes to graded stimulation of supraspinal centers of the brain. J. Neurophysiol. 28: 958-965.

38. Suzuki Y. (1979) Mechanical efficiency of fast- and slow-twitch muscle fibers in man during cycling. J. Appl. Physiol. 47: 263-267.

39. Taylor H. L., Buskirk E., Henschel A. (1955) Maximal oxygen intake as an objective measure of cardio-respiratory performance. J. Appl. Physiol. 8: 73-80.

40. Thomson J. A., Green H., Houston M. E. (1979) Muscle glycogen depletion patterns in fast twitch fibre subgroups of man during submaximal and supramaximal exercise. Pflügers Arch. 379: 105-108.

41. Tissot J. (1904) Nouvelle methode de mesure et d'inscription du debit et des mouvements respiratoires de l'homme et des animaux. J. Physiol. Pathol. Gen. 6: 688-700.

42. Vaage O., Grønnerød O., Dahl H., Hermansen L. (1980) Subgrouping of skeletal muscle fibres in man. Acta Physiol. Scand. 108: $41 \mathrm{~A}$.

43. Vøllestad N. K., Blom P. (1985) Effect of varying exercise intensity on glycogen depletion in human muscle fibres. Acta Physiol. Scand. 125: 395-405.

44. Vøllestad N. K., Vaage O., Hermansen L. (1984) Muscle glycogen depletion patterns in type I and subgroups of type II fibres during prolonged severe exercise in man. Acta Physiol. Scand. 122: 433441.

45. Weisberg S. (1985) Applied Linear Regression. John Wiley \& Sons, New York. 279-281, ISBN 0-471-87957-6.

46. Wendt I. R., Gibbs C. L. (1973) Energy production of rat extensor digitorum longus muscle. Am. J. Physiol. 224: 1081-1086.

\section{Correspondence to:}

Jon Ingulf Medbø

National Institute of Occupational Health

Box 8149 Dep.

NO-0033 Oslo

Norway 